\section{Respiratory Effort Related Arousal}

Helga Peter

Marburg, Deutschland

\section{Synonyme}

RERA

\section{Definition}

Bezeichnung für atmungsbedingte schlaffragmentierende >Arousal“, die bei manchen Patienten mit obstruktiver Schlafapnoe auftreten. RERAs können bei der Bewertung von Befunden aus der Kardiorespiratorischen Polysomnographie gleichberechtigt mit obstruktiven Hypopnoen und Apnoen eingesetzt werden: Mindestens fünf von einem der drei genannten Ereignisse oder die Summe aus allen drei Formen je Stunde Schlafzeit gelten bei einem symptomatischen Patienten (Tagesschläfrigkeit, Insomnie, kardiovaskuläre Erkrankungen) als Mindestkriterium für die Diagnose Obstruktive Schlafapnoe.

\section{Grundlagen}

RERAs haben den identischen Pathomechanismus wie obstruktive Hypopnoen und Apnoen. Die Werte für die Einschränkungen des Luftflusses an Nase und Mund bleiben aber unter $30 \%$ des Ausgangswertes, sodass weder die Messkriterien für obstruktive Hypopnoen noch für Apnoen erfüllt werden. Die zugrunde liegende (partielle) pharyngeale Obstruktion ist jedoch ausreichend, um eine Zunahme der Atmungsanstrengung (Effort) $\mathrm{zu}$ bewirken, in deren Folge wie bei obstruktiven Hypopnoen und Apnoen schlaffragmentierende zentralnervöse Arousals ausgelöst werden. Bei RERAs besteht somit lediglich ein quantitativer Unterschied, bezogen auf den Schwellenwert für die Einschränkung des Luftflusses, der die Reaktionskette von pharyngealer Obstruktion, vermehrtem respiratorischem Effort gefolgt von einem Arousal auslöst.

Bis 2012 konnten Ereignisse mit respiratorisch getriggerten Arousals, aber ohne pulsoxymetrische Sauerstoffdesaturationen von mindestens 3 \% nur als Respiratory Effort Related Arousal (RERA) klassifiziert werden, aber noch nicht als Hypopnoen. Die Definition der Hypopnoe war an eine Sauerstoffdesaturation und eine Atemflusseinschänkung von mindestens $50 \%$ geknüpft. Seit der Hypopnoe-Definition des AASM-Manuals von 2012 können Atmungsstörungen mit Atemflusslimitationen von mindestens $30 \%$ auch unabhängig von Sauerstoffdesaturationen als Hypopnoen gewertet werden, sofern sie durch ein Arousal terminiert werden. Dadurch wurde ein Großteil der RERAs in die Definition der Hypopnoe aufgenommen. Ist bei entsprechenden Ereignissen die Atemflusslimitation geringer als $30 \%$, liegt weiterhin ein RERA vor.

Siehe auch

- >Atmungsmessung“

- „Diagnostik der Schlafbezogenen Atmungsstörungen“

- „Gestörter Schlaf, seine Muster in der Kardiorespiratorischen Polysomnographie“"

- „Obstruktive Schlafapnoe“

- „Schlafbezogene Atmungsstörungen“

- $>$,Schnarchen“" 